\title{
Predictions of projectile penetration and perforation by DAFL with the free surface effect
}

\author{
Q. Fang, X. Kong, H. Wu \& H. Yan \\ Engineering Institute of Engineering Corps, \\ PLA University of Science and Technology, Nanjing, China
}

\begin{abstract}
In this paper, we propose an analytical method to predict projectile penetration and perforation based on the Differential Area Force Law (DAFL) and semiempirical resistance function. Due to the free surface effect, in order to account for the differences of the target resistances acting on the top and bottom sides of the projectile, we obtain the theoretic solutions of a dynamically expanding spherical cavity in a finite sphere of incompressible Mohr-Coulomb target material. The proposed methodology is implemented in ABAQUS explicit solver via the user subroutine. The numerical predictions are in good agreement with the normal and oblique penetration/perforation tests data.

Keywords: penetration, perforation, DAFL, free surface effect, spherical cavity expansion.
\end{abstract}

\section{Introduction}

The general subject of the penetration and perforation of various media by projectiles has recently been surveyed by Forrestal and Luk [1], Hanchak et al. [2], and Frew et al. [3]. Typically, penetration and perforation problems are solved by either empirical, analytical, or numerical methods. In general, empirical methods are mostly employed when the terminal ballistic parameters are desired. Furthermore, empirical methods are the most successful when the penetrator can be approximated as a rigid body. If the structural response of projectiles during penetration is the main concern, analytical or numerical approaches have shown more success. 
Traditional numerical approaches to predict penetration have the firm theoretical basis (based on different representations of the conservation laws for a continuum: Lagrangian, Eulerian, Arbitrary Lagrangian-Eulerian, etc.). With these both the target and the projectile are discretized and integrated numerically in both space and time. The shortcomings of the traditional numerical analysis method are that the sliding surface algorithms are required to define the projectile/target boundary. Computational techniques such as rezoning are usually required to handle the large deformation of elements located in the target. In addition, constitutive models currently available are not adequate to accurately model many targets materials, and Lagrangian approaches often suffer from mesh entanglement in the target that can prematurely terminate the analysis [4]. Also, since this approach requires that the target and the projectile be modeled, these problems generally involve very large number of elements and can be very expensive and time consuming.

Warren and Tabbara [5] and Warren [6] employed PRONTO3D [7] developed by Sandia National Laboratory to simulate the experiments in which ogival-nosed 4340 steel projectiles penetrated 6061-T6511 aluminum and limestone targets at oblique with pitch and yaw. They use an explicit transient dynamic finite element code to model the projectile and an analytical forcing function derived from the dynamic expansion of a spherical cavity to represent the target. This methodology eliminates the need for discretizing the target as well as the need for a contact algorithm, which reduces the computer time and memory requirements. In their study, good agreement was obtained for projectile deformation and final depth of penetration.

However, there must be some correction when the dynamic expansion of a spherical cavity applied to the finite target because it is based on the infinite target. Macek and Duffey [8] used a spherical cavity expansion forcing function derived from a cavity of finite size to account for near-surface effects in the penetration of geologic media. With their forcing function they consider the target to be an incompressible and damaged Mohr-Coulomb material, they point out that it is essential to assume incompressibility because without this assumption time dependent wave propagation and reflections would need to be tracked relative to the moving penetrator. However, the use of a fully incompressible forcing function will cause an over prediction of the target resistance [9]. An alternative method to address free surface effects has been proposed by Warren and Tabbara [5], they constructed a decay function that is based on the solution of a dynamically expanding spherical cavity in a finite sphere of target material which is assumed to be incompressible and perfectly plastic, and then multiply the cavity expansion pressure by the decay function in order to account for the free surface effects. Good agreement was obtained by comparing the final predicted projectile configurations with the post-test radiographs.

The differential area force law (DAFL) method is proposed by the AVCO Corporation in $1970 \mathrm{~s}$, assuming that the resistance of the projectile surface during penetrating can be written in the form of a quadratic function of speed [10]. The US Army Corps of Engineers Waterways Experiment Station (WES) 
has improved DAFL for trajectory analyses [11]. Recently, Li [12] used DAFL and semi-empirical resistance function to formulate the motion of the hard projectile and trajectory stability.

In this paper, a general $3 \mathrm{D}$ model for deformable projectile dynamics is presented, which will then be used to analyze oblique penetration and perforation. In Section 2, we assume the target to be incompressible and MohrCoulomb material and achieve the pressure on the projectile surface with the free surface effect based on the spherical cavity expansion. In Section 3, we briefly describe the framework of deformable projectile penetration with the free surface effect based on DAFL and spherical cavity expansion. This framework is validated in Section 4 and in Section 5 provides conclusive remarks.

\section{Spherical cavity expansion model with the free surface effect}

In this section, we develop an expression that approximates the target response resulted from a dynamic, spherically symmetric cavity-expansion problem. This involves obtaining a decay function that is based on the solution of a dynamically expanding spherical cavity in a finite sphere of a target which is assumed to be incompressible and Mohr-Coulomb material, and then multiply the cavity expansion pressure proposed by Forrestal et al. [13] by the above decay function in order to account for the free surface effect.

\subsection{Spherical cavity expansion in infinite media}

Post-test observations for both concrete and rock targets [3, 13] indicate that there are two regions associated with the penetration of brittle materials as illustrated in Figure 1. The first region is a conical cratering region that is generally about two projectile diameters in depth $\left(H_{c}=2 D\right.$, where $D$ is the diameter of the projectile shank). This is followed by a second tunneling region that is approximately one projectile diameter which starts at the end of the cratering region and proceeds to the final depth of penetration $H_{p e n}$. The normal stress $\sigma_{n}$ acting at the projectile-target interface in the tunneling region is [13]:

$$
\sigma_{n}=S f_{c}+\rho v_{n}^{2}
$$

where $v_{\mathrm{n}}$ is the velocity component normal to the surface of the projectile, $S$ is an empirical strength parameter expressed by $S=82.6 \times\left(f_{c} \times 10^{-6}\right)^{-0.544}[14,15]$ and $S=72 \times\left(f_{c} \times 10^{-6}\right)^{-0.5}[16]$, respectively.

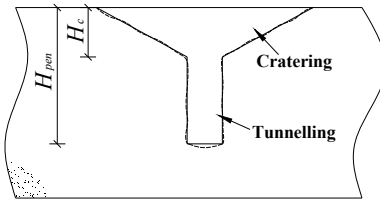

Figure 1: Two-stage penetration model. 
Forrestal et al. [13] treated the cratering region by allowing the target resistance to increase linearly. For the computational method, the cratering region was treated by the technique that the cratering region is divided into 10 uniformly spaced layers with increasing strength. Thus, the normal stress acting at the projectile for $i$ th specific layer $\sigma_{n, i}$ is given by

$$
\sigma_{n, i}=0.1 i \sigma_{n}(i=1,10)
$$

In the cratering region the normal stresses are only allowed to act on the nose of the projectile and not the shank. This accounts for the target material that is ejected out during the initial entry and creates the conical crater [6]. Stresses are then applied on the shank after it enters the tunneling region where the target material is confined and cannot be ejected out.

\section{Spherical cavity expansion with the free surface effect}

Eqn. (1) was acquired based on the infinite dynamically expanding spherical cavity, and we correct it by the method proposed by Warren and Tabbara [5], which construct a decay function that is based on the solution of a dynamically expanding spherical cavity in a finite sphere of a target which is assumed to be incompressible and Mohr-Coulomb material.

To obtain the decay function we consider the expansion of a spherically symmetric cavity from zero initial radius to radius $a$, this expansion produces plastic and elastic response regions, as shown in Figure 2.

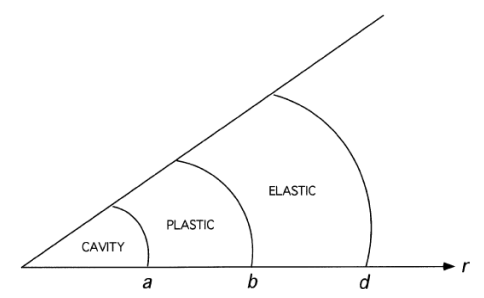

Figure 2: Response regions for the cavity expansion problem.

The equations of momentum and mass conservation in Eulerian coordinates with spherical symmetry are

$$
\begin{gathered}
\frac{\partial \sigma_{r}}{\partial r}+\frac{2\left(\sigma_{r}-\sigma_{\theta}\right)}{r}=-\rho\left(\frac{\partial v}{\partial t}+v \frac{\partial v}{\partial r}\right) \\
\rho_{0} \frac{\partial}{\partial r}\left[(r-u)^{3}\right]=3 \rho r^{2}
\end{gathered}
$$

where $\sigma_{r}, \sigma_{\theta}$ are the radial and hoop components of the Cauchy stress, and $\rho_{0}$ and $\rho$ are the densities in the undeformed and deformed states. $\rho=\rho_{0}$ when the 
material is assumed incompressible. Particle displacement $u$ and particle velocity $v$ in the radial direction are related by

$$
\frac{\partial u}{\partial t}=v\left(1-\frac{\partial u}{\partial r}\right)
$$

The material in the plastic region is described by an incompressible MohrCoulomb yield criterion, thus:

$$
\begin{gathered}
p=\left(\sigma_{r}+\sigma_{\theta}+\sigma_{\phi}\right) / 3, \sigma_{\theta}=\sigma_{\phi} \\
\sigma_{r}-\sigma_{\theta}=\lambda p+\tau
\end{gathered}
$$

where $p$ is the pressure, and $\lambda$ and $\tau$ denote the pressure-dependent shear strength parameters, respectively.

Solving the eqns. (1)-(6) and considering the continuum of the radial stress on the interface $r=b$ and $r=d[6,17]$, the radial stress at the cavity $\sigma_{r}(a)$ can be obtained as follows.

$$
\left\{\begin{array}{l}
\sigma_{r}(a)=\left\{2 \rho \dot{a}^{2}\left[\frac{1}{\alpha \lambda-4}-\frac{1}{\alpha \lambda-1}\right]-\frac{\tau}{\lambda}+\left(\frac{2 E}{3 \tau}\right)^{\alpha \lambda / 3}\left\{\frac{2 \tau}{3}\left[1-\left(\frac{b}{d}\right)^{3}\right]\right\},\right. \\
\left.+2 \rho \dot{a}^{2}\left[\frac{\alpha \lambda}{\alpha \lambda-1}\left(\frac{3 \tau}{2 E}\right)^{1 / 3}-\frac{1}{4}\left(\frac{\alpha \lambda}{\alpha \lambda-4}\right)\left(\frac{3 \tau}{2 E}\right)^{4 / 3}-\frac{a}{d}+\frac{1}{4}\left(\frac{a}{d}\right)^{4}\right]+\frac{\tau}{\lambda}\right\} . \quad d \geq b \\
\sigma_{r}(a)=2 \rho \dot{a}^{2}\left(\frac{1}{\alpha \lambda-4}-\frac{1}{\alpha \lambda-1}\right)-\frac{\tau}{\lambda}+\left(\frac{b}{a}\right)^{\alpha \lambda}\left\{2 \rho \dot { a } ^ { 2 } \left[\frac{\alpha \lambda}{\alpha \lambda-1}\left(\frac{a}{b}\right),\right.\right. \\
\left.\left.-\frac{1}{4}\left(\frac{\alpha \lambda}{\alpha \lambda-4}\right)\left(\frac{a}{b}\right)^{4}-\left(\frac{a}{b}\right)+\frac{1}{4}\left(\frac{a}{b}\right)^{4}\right]+\frac{\tau}{\lambda}\right\} . \quad d<b
\end{array}\right.
$$

where $\dot{a}$ is the cavity expansion velocity, and the cavity radius $a$ is only a function of time, $\alpha=6 /(3+2 \lambda), E$ is the elastic modulus.

We now have constructed a decay function, which allows us to employ the results from eqn. (7) with the expression given in eqn. (1), (2) that accounts for the free surface effect. This is done by normalizing eqn. (7) with the result given by eqn. (7) letting $d \rightarrow \infty$. For brittle materials the plastic zone actually consists of granular phase material so we assume that no resistance to penetration is provided once the plastic zone reaches the free surface. Thus,

$$
f(d, a, \dot{a})= \begin{cases}\frac{\sigma_{r}(a)}{\sigma_{r}(a)_{d \rightarrow \infty}}, & d \geq b \\ 0, & d<b\end{cases}
$$

Plots of the normalized radial stress as a function of $d / a$ obtained from eqn. (7) for several expansion velocities are shown in Figure 3. The parameters used in eqn. (7) for unconfined compressive strength 48MPa concrete are summarized in Table 1. It is observed that free surface effects extend to larger distances as the expansion velocity is increased. 
Table 1: $\quad$ Parameter of 48MPa unconfined compressive strength concrete.

\begin{tabular}{|c|c|c|c|c|c|}
\hline$S f_{\mathrm{c}}$ & $E$ & $Y$ & $\rho_{0}$ & $\lambda$ & $\tau$ \\
\hline $500 \mathrm{MPa}$ & $37 \mathrm{GPa}$ & $48 \mathrm{MPa}$ & $2440 \mathrm{Kg} / \mathrm{m} 3$ & 0.52 & $46 \mathrm{MPa}$ \\
\hline
\end{tabular}

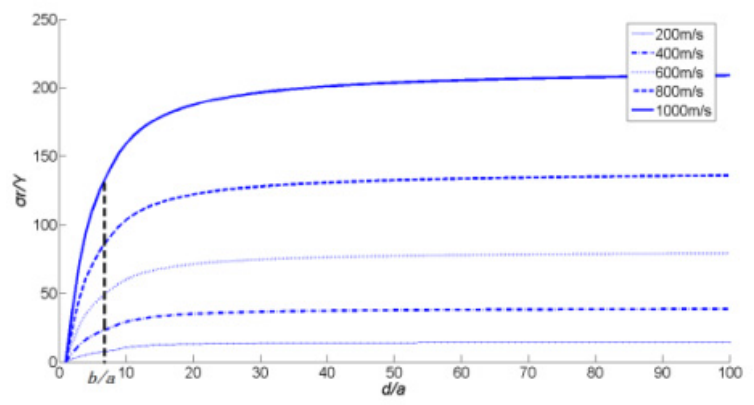

Figure 3: Radial stress at the cavity surface vs. distance to the free surface.

Eqn. (7) allows Forrestal's semi-empirical model to account for a finite boundary. It must be noted that there are two free-surfaces should be taken into account if the target is finite thickness, as shown in Figure 4. Thus, the normalized radial stress on the projectile surface with surface effect gives as follow:

$$
\sigma_{n}=\sigma_{r}(a)=\left\{\begin{array}{cc}
f(d, a, \dot{a}) \times 0.1 i \times\left(S f_{c}+\rho v_{n}^{2}\right), & H_{p e n} \leq 2 D \\
f(d, a, \dot{a}) \times\left(S f_{c}+\rho v_{n}^{2}\right), & H_{p e n}>2 D
\end{array}\right.
$$

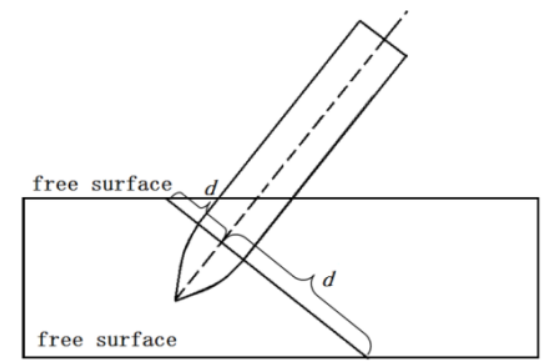

Figure 4: $\quad$ Finite target with two free surfaces.

\section{Implementation of the proposed analytical method}

Based on the analysis of the previous section, the target was represented by an analytical forcing function derived from the dynamic spherical expansion cavity during the penetration process. This methodology eliminates the need for 


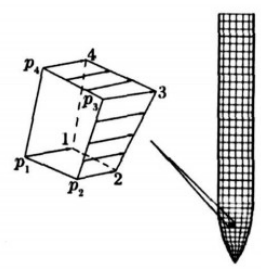

Figure 5: Definition of a pressure boundary condition.

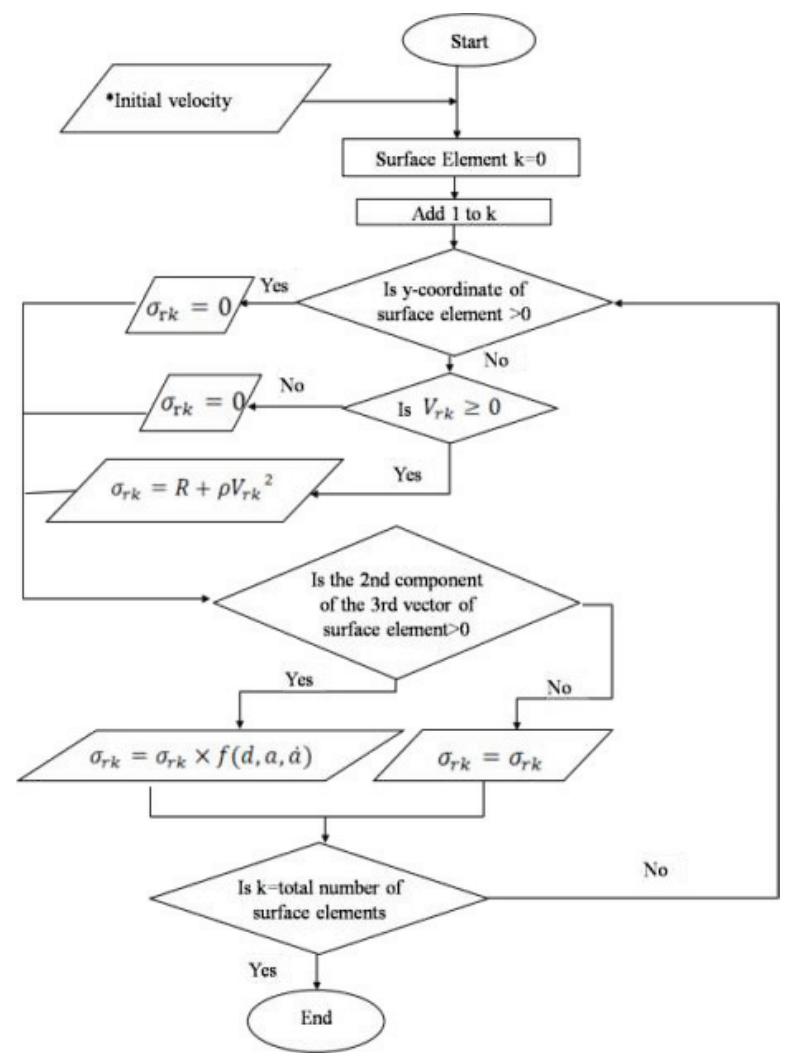

Figure 6: $\quad$ Flow chart of the algorithm for the proposed method.

discretizing the target as well as the need for a contact algorithm, which reduces the computer time and memory requirements. The pressure on the elements of the projectile surface is shown in Figure 5 and the pressure on each nodal can be expressed as:

$$
P=\sigma_{n}= \begin{cases}f(d, a, \dot{a}) \times 0.1 i \times\left(S f_{c}+\rho(\boldsymbol{v} \bullet \boldsymbol{n})^{2}\right), & H_{p e n} \leq 2 D \\ f(d, a, \dot{a}) \times\left(S f_{c}+\rho(\boldsymbol{v} \bullet \boldsymbol{n})^{2}\right), & H_{p e n}>2 D\end{cases}
$$


where $\boldsymbol{v}$ is the nodal velocity vector, $\boldsymbol{n}$ is the outward unit vector normal to the element surface. The values of $P$ are updated during each time increment using the current values of $\boldsymbol{v}$ and $\boldsymbol{n}$. When the projectile hit the target with oblique angle, the contact and separation between the projectile and target must be considered. Note that eqn. (10) is used only if $(\boldsymbol{v} \bullet \boldsymbol{n})$ at a node is larger than zero, and zero is set if $(\boldsymbol{v} \bullet \boldsymbol{n})$ at a node is negative.

The framework of deformable projectile penetration introduced above was implemented in ABAQUS explicit solver via the user subroutine, which is used to define a distributed pressure on the outer surface of the projectile, both the nose and the shank. The subroutine is called in each time step and calculates the pressure to be applied on the integration point $i$ where the distributed load is defined. Figure 6 shows a flowchart of the algorithm coded in Fortran. The process is repeated until all surface elements of the projectile are processed by the user subroutine. The reader is referred to the ABAQUS Analysis User's Manual [18] for further information of user subroutine.

\section{Analyses and discussions}

\subsection{Normal depth of penetration}

Figure 7 shows the predicted penetration depth of the normal penetration tests [13]. It indicates that, the predicted results agree well with the experimental results in the velocity of less than $820 \mathrm{~m} / \mathrm{s}$ and about $10 \%$ underestimation of the experimental result in the velocity of $1000 \mathrm{~m} / \mathrm{s}$ is observed. The underestimation may be attributes to the deviations of the resistance function in high speed penetration.

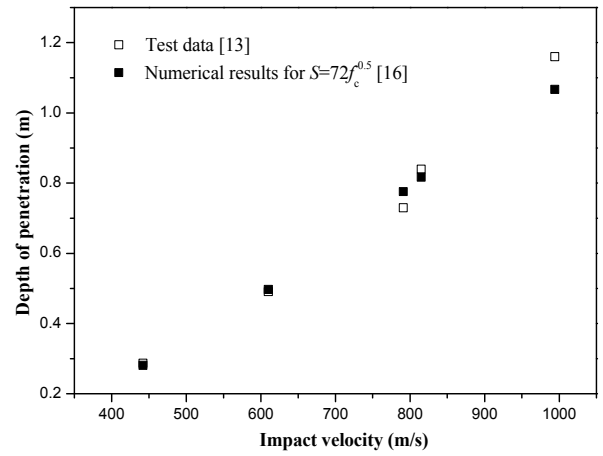

Figure 7: Comparisons between the experimental results and numerical simulations for concrete targets.

\subsection{Verification of the free surface effects in oblique penetration}

With larger angles of obliquity, the resistance force on the side of the projectile close to the free surface will be affected. The decay function for free surface 

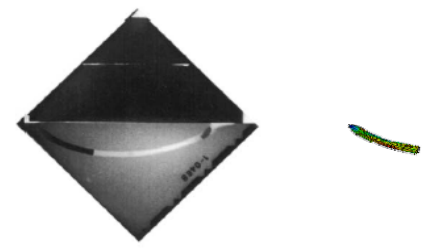

(a) experiment (b) with free surface effect (c) without free surface effect

Figure 8: Comparison between experimental and numerical simulations for aluminum target.

effects is shown in eqn. (8). Figure 8 gives the experimental projectile trajectory [5] as well as the simulated results of the trajectory and final configuration of the projectile, while the free surface effects were considered (Figure $8(\mathrm{~b})$ ) or not (Figure 8(c)). It is observed that, when the free surface effect is included, the simulation provides a very reasonable prediction of the trajectory and final configuration of the projectile.

\subsection{Perforation of concrete slabs}

We simulate the perforation of concrete slabs with $48 \mathrm{MPa}$ and $140 \mathrm{MPa}$ unconfined compressive strengths, and compare the predicted residual velocities with experimental data [2]. The effect of the rear surface of the target plate was considered. Figure 9(a) shows that the predictions agree well with the experiment results for concrete slab with $48 \mathrm{MPa}$ unconfined compressive strength. But for concrete slab with $140 \mathrm{MPa}$ unconfined compressive strength and the parameter $S$ recommended by $\mathrm{Li}$ and Chen [16], the predictions do not agree well. It is because the parameter $S$ recommended by Li is not suit for highstrength concrete. If we use the parameter recommended by $\mathrm{Wu}$ et al. [19], a good agreement between the predictions and the experiment results is observed.

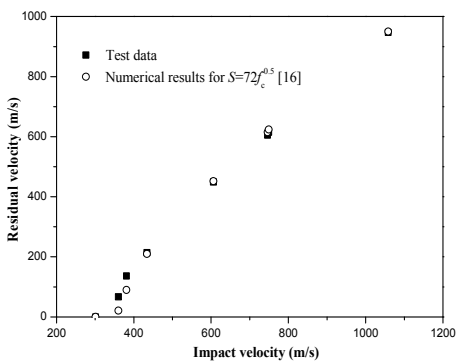

(a) $48 \mathrm{MPa}$

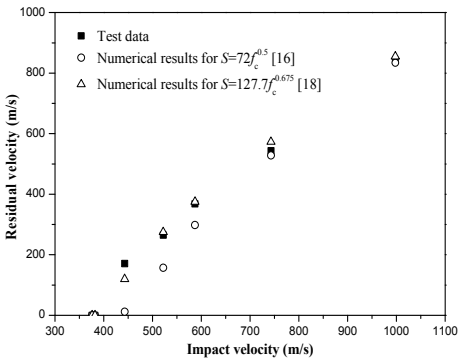

(b) $140 \mathrm{MPa}$

Figure 9: Comparison between experimental results and numerical simulation for perforation of concrete slab. 


\section{Conclusion}

In this paper, a numerical method, in which the free surface effects are considered is proposed due to angle of obliquity, is proposed to predict projectile penetration and perforation based on the Differential Area Force Law (DAFL) and semi-empirical resistance function. The methodology is implemented in ABAQUS explicit solver via the user subroutine. Based on the comparisons between the predicted results with the vertical penetration and perforation tests data on normal strength and high strength concrete targets as well as the oblique penetration tests data on aluminum target. The validity of the proposed method is verified. The computational efficiency of this method is also much higher than other methods such as finite element method. It is indicated that proposed method represents a very effective method of determining the response of the projectiles in penetration and perforation.

\section{Acknowledgements}

The project was supported by the Funds for Creative Research Groups of China (51021001), and the National Natural Science Foundations of China (51008304, 51178461).

\section{References}

[1] Forrestal, M.J. and Luk, V.K., Penetration into soil targets. International Journal of Impact Engineering, 12, pp. 427-444, 1992.

[2] Hanchak, S.J., Forrestal, M.J. and Young, E.R., Perforation of concrete slabs with $48 \mathrm{MPa}$ and $140 \mathrm{MPa}$ unconfined compressive strength. International Journal of Impact Engineering, 12(1), pp. 1-7, 1992.

[3] Frew, D.J., Forrestal, M.J. and Hanchak, S.J., Penetration Experiments With Limestone Targets and Ogive-Nose Steel Projectiles. Journal of Applied Mechanics, 67, pp. 841-845, 2000.

[4] Camacho, G.T. and Ortiz, M., Adaptive Lagrangian modeling of ballistic penetration of metallic targets. Computer Methods in Applied Mechanics and Engineering, 142, pp. 269-301, 1997.

[5] Warren, T.L. and Tabbara, M.R., Simulations of the penetration of 6061T651l aluminum targets by spherical-nosed VAR4340 steel projectiles. International Journal of Solids and Structures, 37(44), pp. 4419-4435, 2000.

[6] Warren, T.L., Simulations of the penetration of limestone targets by ogive-nose 4340 steel projectiles. International Journal of Impact Engineering, 27, pp. 475-496, 2002.

[7] Taylor, L.M. and Flanagan, D.P., PRONTO3D a three-dimensional transient solid dynamics program. SAND87-1912, Sandia National Laboratories, Albuquerque, NM, 1989. 
[8] Macek, R.W. and Duffey, T.A., Finite cavity expansion method for nearsurface effects and layering during earth penetration. International Journal of Impact Engineering, 24, pp. 239-58, 2000.

[9] Warren, T.L. and Forrestal, M.J., Effects of strain hardening and strainrate sensitivity on the penetration of aluminum targets with sphericalnosed rods. International Journal of Solids and Structures, 35, pp. 373753, 1998.

[10] Adeli, H., Amin A. M. and Sierakowski, R.L., Earth penetration by solid impactors. Shock Vibration Digest, 18, pp. 15-22, 1986.

[11] Bernard, R.S. and Creighton, D., Projectile penetration in soil and rock: analysis for non-normal impact, Technical Report .SL-79-15AD-A081044, US Army Waterways Experiment Station, Vieksburg, 1979.

[12] Li, Q.M. and Flores-Johnson, E.A., Hard projectile penetration and trajectory stability. International Journal of Impact Engineering, 38(1), pp. 815-823, 2011.

[13] Forrestal, M.J., Altman, B.S., Cargile, J.D. and Hanchak, S.J., An empirical equation for penetration depth of ogive-nose projectiles into concrete targets. International Journal of Impact Engineering, 15(4), pp. 395-405, 1994.

[14] Forrestal, M.J., Frew, D.J. and Hanchak S.J., Penetration of grout and concrete targets with ogive-nose steel projectiles. International Journal of Impact Engineering, 18(5), pp. 465-476, 1996.

[15] Frew, D.J., Hanchak, S.J. and Green, M.L., Penetration of concrete targets with ogive-nose steel rods. International Journal of Impact Engineering, 21(6), pp. 489-497, 1998.

[16] Li, Q.M. and Chen, X.W., Dimensionless formulae for penetration depth of concrete targets impacted by rigid projectiles. International Journal of Impact Engineering, 28, pp. 93-116, 2003.

[17] Hopkins, H.G., Dynamic expansion of spherical cavities in metals. Progress in solid mechanics, 1. pp. 5-164, 1960

[18] ABAQUS. Analysis user's manual, Version 6.5, 2004.

[19] Wu, H., Fang, Q. and Gong, Z.M., Experimental and engineering analyses on DOP of HSFRC target under the impact of rigid projectile. Journal of Ballistics, Accepted (in Chinese). 